\title{
Stress Prediction and Hydrodynamics from Multicomponent Seismic Data
}

Wildney W. S. Vieira, Lourenildo W. B. Leite and Boris P. Sibiryakov, UFPA, Brazil

Copyright 2014, SBGf - Sociedade Brasileira de Geofísica.

This paper was prepared for presentation at the VI Brazilian Symposium on geophysics, held in Porto Alegre, Brazil, October 14-16, 2014. Contents of this paper were reviewed by the Technical Committee of the VI SimBGf and do not necessarily represent any position of the SBGf, its officers or members. Electronic reproduction or storage of any part of this paper for commercial purposes without the written consent of The Brazilian Geophysical Society is prohibited.

\section{Abstract}

This paper treats a proposed theory and application to detect and outline oil fields, and divide them into isolated traps marked by the low overburden pressure and fluidtrapping properties based on a correlation between the structural pattern and mapped stress field.

Unlike the traditional methods of hydrodynamic modeling, the new method is based on 3D multicomponent seismic exploration data $\left(V_{\mathrm{p}}, V_{\mathrm{s}}, \rho\right)$.

We also focus in the so much attention that has been recently given to stress predicition, shear waves, porosity, specific surface, pressure, and 3D hydrodynamic modeling of buried oil and gas traps.

A different systematic approach implies the use of seismic data $\left(V_{\mathrm{p}}\right.$ and $V_{\mathrm{s}}$ velocities and density $\rho$ ) for stress modeling of reservoirs, in order to detect regions of low overburden pressure $P(x, y, z)$, and vertical inclination of fractures (faults) $\varphi(x, y, z)$ caused by the nonhydrostatic behavior of stress.

\section{Introduction}

Sibiryakov (2002) presented theory and appplications on pore space and integral geometry. And as a rule, for small porosity there is a large specific surface area that creates anomalous high $\gamma=\frac{v_{S}}{v_{P}}$ ratio, what can produce a negative Poisson coefficient, $\sigma=\frac{1-2 \gamma^{2}}{2-2 \gamma^{2}}$.

The state of elastic layered media is described in the general case by six (three normal and three tangential) stress tensor components. Complex stress behavior of buried traps is modeled on the basis of $P$ and $S$ layer velocities, and of the bulk density of reservoir and cap rocks. Overburden pressure and tangential stress intensity (the measure of nonhydrostatic behavior of stress) are other key parameters, the so-called scalar invariants of stress tensor. (Sibiryakov, 2004).

Stress is nonhydrostatic even in horizontal layered media subject to only vertical gravity compactation without horizontal displacement. Vertical stress is defined as equal to the weight of the overburden in the form: $\sigma_{z z}=P_{z}=P_{0}$. The correspondent horizontal stress is sufficiently lower, and given by: $\sigma_{x x}=P_{x}=P_{0}\left(1-2 \gamma^{2}\right),\left(\sigma_{x x}=\sigma_{y y}\right.$ in this case $)$, than $P_{z}$, where $\gamma=\frac{V_{S}}{V_{P}}$. (Sibiryakov et al., 2004).

Pressure is always a scalar quantity, and the simplest case for overburden pressure is given by $P=\frac{\sigma_{x x}+\sigma_{y y}+\sigma_{z z}}{3}=P_{0}(1-$ $\left.\frac{4}{3} \gamma^{2}\right)$, which is sufficiently less than the overlying weight $P_{z}$. (Sibiryakov et al., 2004).

The overburden pressure breaks at layer boundaries if the velocity ratio $\gamma$ has a discontinuity. In the simplest case of layered media, the jump of overburden pressure at layer boundaries is $\Delta P=\frac{4}{3} P_{0}\left(\gamma_{1}^{2}-\gamma_{2}^{2}\right)$ (Sibiryakov et al., 2004). This jumpwise characteristics (positive and negative) can appear strange to geologists, but it is a fact related to the nonelementary behavior of stress in solids.

The intensity of tangential stress is a measure of the mechanical instability, and it is the parameter responsible for the failure of the solid rock skeleton and fracturing. In simple layered media, the tangential stress, scalar invariant, is given by $P_{T}=J=\frac{\sigma_{z z}-\sigma_{x x}}{2}$, it also depends on the velocity $\gamma$ ratio, and it is shown to be given by: $J=\gamma^{2} P_{z}$ (Sibiryakov et al., 2004).

\section{Methodology}

\section{Example}

Sibiryakov and Zaikin (1994) have applied this method for 2D applications to stress modeling in seismic sections in East and West Siberia. But, the direct extrapolation of the method to 3D problems complicates the computation.

Referring to Figure 1, the overburden pressure and intensity of tangential stress estimated on the basis of the $\gamma$ velocity ratio were assumed constant over the reservoir, and the flow lines are thus temptative. Almost all lines converge toward the top of the structure. The points of local discharge, to the right and left of the main borehole complex, are prominent even in this generalized model.

Figure 2 shows the map of tangential stress intensity for the same surface of Figure 1, where the region of lowest pressure almost coincides with faults within the region of minimum tangential stress, which indicates a certain stability of the structure as a whole. High tangential stresses cluster near a fault, which appears as a rather realistic result.

Figure 3 shows calculated fracture inclinations, $\phi(x, y, z)$, around the structure. The fracture planes are inclined about 45 degrees with respect to the vertical, which may correspond to either randomly oriented fractures or to anisotropic medium with rhombic symmetry. However, fractures change their inclinations near faults to roughly vertical inclinations, thus producing another preferred inclination.

The vertical inclination of fractures, called $\phi(x, y, z)$, are obtained from the rotation of the stress system to give the stress diagonal matrix, where the diagonal elements are the main stresses, and where $\sigma_{x x} \approx \sigma_{y y}$ (Novacky, 1970). 


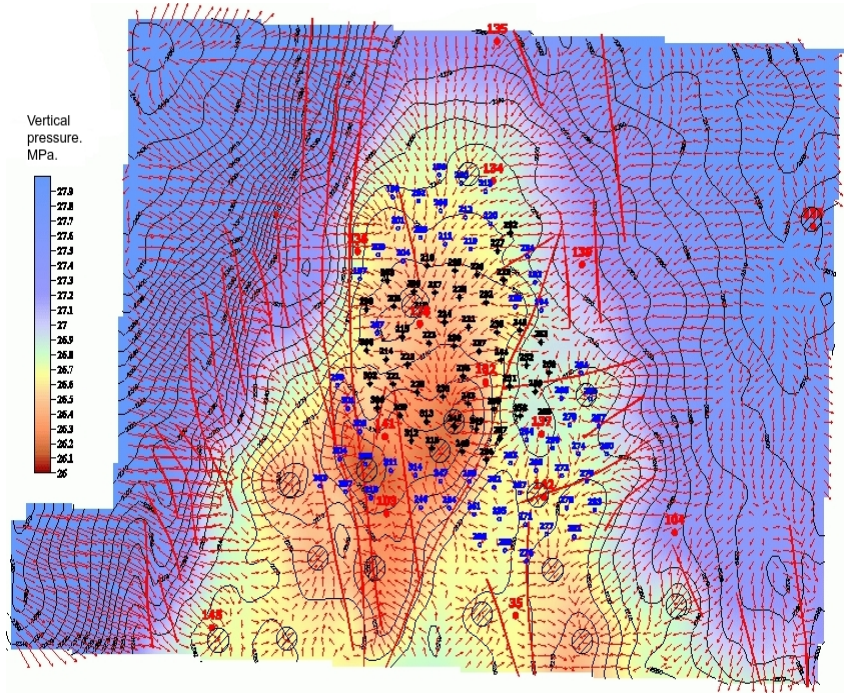

Figure 1: Tectonic stress of Jurassic reservoir where the contour lines are overburden pressure $P=P_{0}\left(1-\frac{4}{3} \gamma^{2}\right)$ (dark blue lines) drawn in an arbitrary scale, and superposed to the color scale. Orthogonal trajectories are probable fluid flow lines (red arrows). The pressure scale is in color and placed on the left side, and shows variations between 26-28 Mega-Pascal (MPa), $1 \mathrm{MPa}=10$ Atmospheres. The center of the map is dominated by a low pressure zone. The dots with numbers are productive boreholes. Heavy red lines are geological faults. Isolated circular enclaves correspond to local hydrodynamic systems.

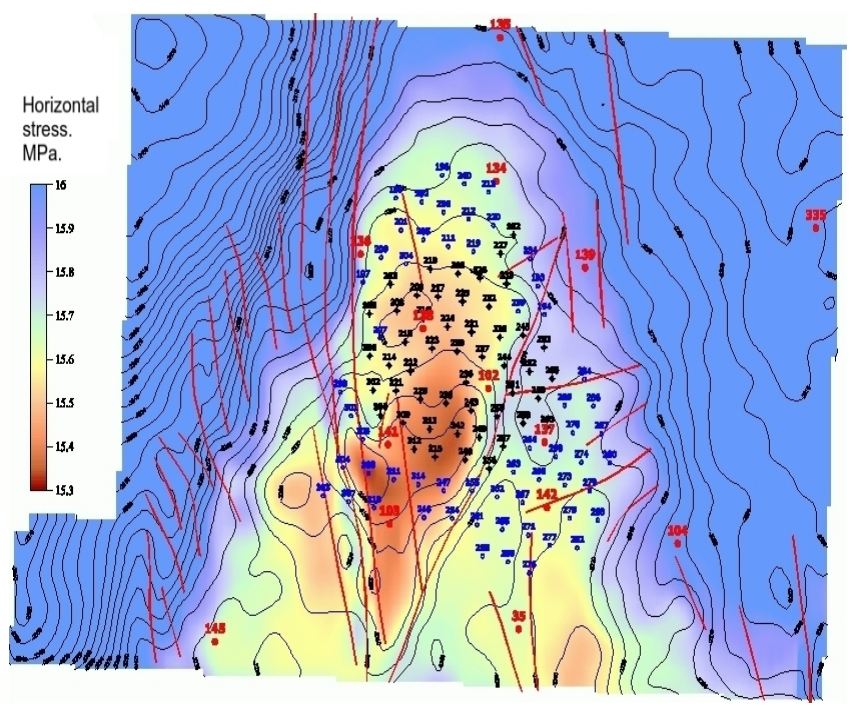

Figure 2: Intensity of tangent stress, $J=\gamma^{2} P_{0}$, as a measure of nonhydrostatic pattern (dark blue lines), where the high values are located around small stress zones. The lines are drawn in an arbitrary scale, and superposed to the color scale. The color pressure scale is on the left side and shows values between 15.3-16.0 in MPa. The dots with numbers are productive wells. Heavy red lines are geological faults.

\section{Physics, the Stress Tensor and Its Invariants}

The system of equations of equilibrium used to estimate stresses is given below, where two differential equations

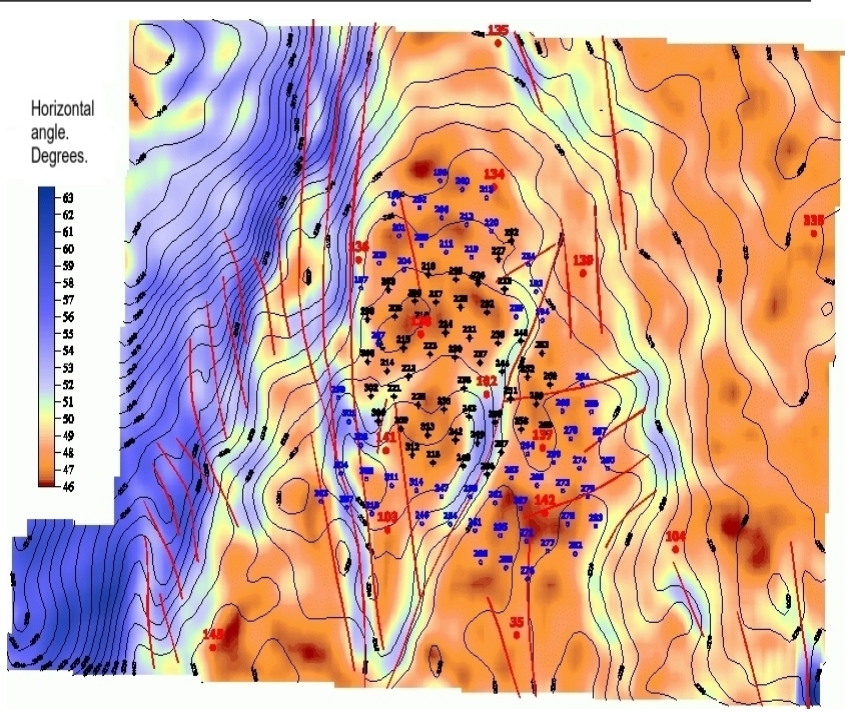

Figure 3: Calculated fracture inclinations $\phi(x, y, z)$ in producing bed. The color scale of the left shows a variation between 46 and 64 degrees with respect to the vertical (to the left and/or to the right). The blue areas shows roughly vertical fractures, and the brown areas show more Normal fractures with a more chaotic distribution. In the center of the map, we can describe more randomly inclined fractures (darker areas). The dots with numbers are productive wells. Heavy red lines are geological faults.

are homogeneous and one nonhomogeneous (Sibiryakov and Prilous, 2007):

$$
\begin{aligned}
& \frac{\partial \sigma_{x x}}{\partial x}+\frac{\partial \sigma_{x y}}{\partial y}+\frac{\partial \sigma_{x z}}{\partial z}=0 \\
& \frac{\partial \sigma_{y x}}{\partial x}+\frac{\partial \sigma_{y y}}{\partial y}+\frac{\partial \sigma_{y z}}{\partial z}=0 \\
& \frac{\partial \sigma_{z x}}{\partial x}+\frac{\partial \sigma_{z y}}{\partial y}+\frac{\partial \sigma_{z z}}{\partial z}=\rho g .
\end{aligned}
$$

The first two equations ( 1 and 2 ) correspond to full cancellation of horizontal $3 \mathrm{D}$ forces produced by inner stresses, and the last one means that vertical 3D forces are simply equal to the normalized gravity force.

The fundamental solution of the system (1-2-3) is given by Novacky (1970) in the form:

$$
u_{k}(\mathbf{x})=\frac{g}{V_{S}^{2}} \int_{V} \Gamma_{k z}(\mathbf{x}, \mathbf{y}) d V_{y} .
$$

The additional displacement $u_{k}(x)$ due to structure deformation is the integral over the structure volume $V$ of the fundamental solution, $\Gamma_{k z}(\mathbf{x}, \mathbf{y})$, of the equilibrium equation, it depends on the square S-wave velocity (4), and $z$ in $\Gamma_{k z}$ is related to the vertical component as the gravity $g$. The integration covers all the volume represented by the $\mathbf{y}$ coordinate.

\section{Pressure in rock skeleton and fluid}

For a fluid-filled reservoir, consider $u_{n}$ as the normal displacement of a skeleton grain. Applying the Gauss theorem, we obtain:

$$
\iint_{S} u_{n} d s=\iiint_{V} \operatorname{div} \vec{u} d V=f V \frac{P_{0}}{\rho_{0} c^{2}},
$$


where $S$ is the grain surface, $V$ the total volume, $f V$ is the pore space volume, $P_{0}$ is the fluid pressure, and $\rho_{0} c^{2}$ is the fluid inverse compressibility (noncompressibility). Also, we can write the approximation:

$$
\iint_{S} u_{n} d s=<u_{n}>S,
$$

where $\left\langle u_{n}\right\rangle$ is the mean normal displacement, and by substituting in (5) gives:

$$
\frac{P_{0}}{\rho_{0} c^{2}}=\frac{1}{f} \frac{<u_{n}>}{r_{0}} \sigma_{0} r_{0}
$$

where $\sigma_{0}=\frac{S_{P}}{V_{T}}$ is the specific surface area $\left(S_{P}\right.$ is the total empty porous, and $V_{T}$ is the total volume of sample), and $r_{0}$ is the mean grain radius.

In a solid grain, the relation:

$$
\Delta\left(u_{i}+x_{i} e\right)=0
$$

is a rigorous relationship, where $\Delta$ is the Laplace operator, $u_{i}$ is the displacement in grain, and $e$ is the dilatation of the grain material. By definition, the pressure is given by the dilation times the bulk module, as:

$$
P=e\left(\lambda+\frac{2}{3} \mu\right)
$$

Equation (8) means, that the sum $u_{i}+x_{i} e$ is a harmonic vector. As its mean value is zero in the grain center, we can assume this harmonic function to be zero in the grain material, and thus relate displacement, $u_{i}$, and dilatation, $e$; in other words, $u_{i}=-x_{i} e$, and

$$
<u_{n}>=-<e>r_{0} .
$$

Equation (10) relates normal displacement in liquid and dilatation in solid.

Substituting the relations (10) and (9) into equation (7), we can write in explicit form the pressure discontinuity, $\frac{P_{0}}{P}$, between solid and liquid:

$$
\frac{P_{0}}{P}=\frac{\sigma_{0} r_{0}}{f} \frac{\rho_{0} c^{2}}{\lambda+\frac{2}{3} \mu} .
$$

Equation (11) shows that the pressure gradient in solid, $\nabla P$, perfectly coincides with the fluid flow lines in the pore structure, $\nabla P_{0}$, and it remains invariable in the productive layer, if the structure of pore space (porosity and specific surface) are constant in this layer.

Compressibility decreases abruptly at low gas contents, and viscosity varies slowly. Therefore, gas saturation of fluids is not necessarily favorable to oil production, but reduces the pressure gradient which is not always canceled by the respective fluid viscosity decrease.

According to (10), the problem of calculating fluid flow into a borehole is reduced to the integration of Poisson's equation given by:

$$
\Delta \dot{u}_{i}=\frac{1}{\eta} \frac{\sigma_{0} r_{0}}{f} \frac{\rho_{0} c^{2}}{\lambda+\frac{2}{3} \mu} \operatorname{grad}_{i} P
$$

where $\operatorname{grad}_{i}=\nabla_{i}=\frac{\partial}{\partial x_{i}},(i=x, y, z)$, and $\eta$ the fluid viscosity. Boundary conditions for this equation (conditions of viscous adhesion) require the velocities of particles be zero over the pore surface. The problem of integration of (12) and flow rate estimation requires calculating of stress and, on the other hand, the knowledge of two parameters of the pore space and fluid compressibility.

The methods of integration for equation (12) are straightforward, even in the case of an arbitrarily complex pore structure; permeability is unnecessary as it appears in a complex way as a result of phase interaction and pore structure. Considering a particular case of noninteracting fractures, modeled by rectilinear segments with constant crack opening, equation (12) can be easily integrated to yield an equation for flow rate:

$$
\dot{u}_{i}=\frac{\delta^{2}}{12 \eta} \frac{\sigma_{0} r_{0}}{f} \frac{\rho_{0} c^{2}}{\lambda+\frac{2}{3} \mu} \operatorname{grad}_{i} P=\operatorname{Kgrad}_{i} P,
$$

where $\delta$ is the mean crack opening, and $\delta^{2}$ represents permeability for this particular pore structure. Therefore, the pressure difference between the skeleton and the fluid depends on the geometry of the pore space (mainly on its specific surface), and on the hydrodynamic properties (compressibility and viscosity) of the fluid. The problem of pore pressure estimation cannot be solved separately from the problem of general stress calculation. Equation (13) plays the role of Darcy's law for the specific model now in focus.

\section{Percolation and permeability}

Sibiryakov (2002) described that the estimation of stress and strain in oil-gas structure require the integration of the equations of equilibrium of the form:

$$
\frac{\partial \sigma_{i k}}{\partial x_{k}}=\rho g, \quad(i, j, k=1,2,3 ; \quad \text { or } \quad x, y, z),
$$

for every layer.

For elastic rock deformation, the equation of equilibrium derived from equations (1), (2), and (3) under the condition of Hooke's law in closed form is given by:

$$
(\lambda+\mu) \operatorname{grad}_{i} \operatorname{div} \vec{u}+\mu \Delta u_{i}=\rho g_{i},
$$

where $g_{i},(i=x, y, z), g_{z}=g, g_{x}=g_{y}=0$, and $\Delta=\nabla \cdot \nabla$ is the Laplace operator (Landau, 1988). The Lame's parameters $\lambda$ and $\mu$ are determined from $\mathrm{P}$ and $\mathrm{S}$ wave velocities. As to boundary conditions, we can write the stress for the inside of any layer an expression of the form:

$$
\sigma_{i k} n_{k}^{+}\left(S_{m}\right)=\sigma_{i k} n_{k}^{-}\left(S_{m}\right), \quad u_{i}^{+}\left(S_{m}\right)=u_{i}^{-}\left(S_{m}\right),
$$

where $S_{m}$ stands for a surface numbered $m$, and $n^{+}, n^{-}$ points to outside or to the inside of the surface $S_{m}$.

On the free Earth's surface the boundary conditions for the stress is given by:

$$
\sigma_{i k} n_{k}^{+}\left(S_{0}\right)=0,
$$

where the summation over the repeated index is implicit.

Besides conditions (16) and (17), there are some conditions on the lateral surfaces that bounds the oil-gas structure. In common case these conditions depend on the geological conditions, but often this conditions is not 
sufficiently enough, especially if the horizontal dimension of the structure is larger than vertical dimension.

Sometimes, it is possible to use as boundary conditions the normal field of stresses without the effect of structure. And for this condition, the fluid pressure equals the normal stress in solid matrix, but not the pressure in solid, because the lateral stresses are not equal to normal stress. In order to calculate a discontinuity of pressure between liquid and solid, it is necessary to integrate the equation of equilibrium in a small volume of the productive layer with boundary conditions on pore space like:

$$
\sigma_{i k} n_{k}^{+}(S)=P_{\infty}(S), \quad u_{i}^{+}(S)=u_{i}^{-}(S) .
$$

In the above equation $P_{\infty}=$ constant is the pressure in the fluid. On the outside boundaries of elementary volume (edges of cube, for example) we need require the same displacements like in the edges of elastic cube without pore space. These conditions gives the constant value $P_{\infty}$, which is in equilibrium state with respect to stresses in solid. No doubt that this pressure depends on the structure of pore space, but first of all on the specific surface of pore space.

\section{The volume dissipative forces by viscosity}

The tangential surface force due to viscosity is given by the expression $F_{S \tau}=\sigma_{0} \frac{\partial u_{\tau}}{\partial n}$, while the tangential volume force due to the same reason is given by:

$$
F_{V \tau}=\sigma_{0} \eta \frac{\partial u_{\tau}}{\partial n}
$$

where $\sigma_{0}$ is the specific surface, $\eta$ is viscosity, $\dot{u}$ is particle velocity, and $n$ and $\tau$ are the normal and tangent directions of liquid-grain contact. For cracks sufficiently long compared to their opening, it would be convenient to use a simpler problem from the Navier-Stokes equation; to be specific, the solution of the problem about flowing between two infinite plates in the form (Landau, 1988):

$$
\frac{\partial p}{\partial x}=\eta \frac{\partial^{2} u_{\tau}}{\partial z^{2}}
$$

In this equation $p$ is pressure in liquid, and $u_{\tau}=u_{x}=u_{x}(z)$ in the particular case of Figure 4, where $u_{y}=u_{z}=0$, and it represents a layout of the physical process of the flow, showing the sticking at the boundaries. The solution of

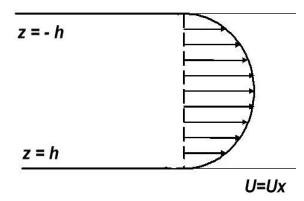

Figure 4: Cross section of flow between infinite two plates. The length of dominant fracture is much larger than the crack opening.

equation (20) takes the form:

$$
\frac{\partial p}{\partial x}=P=\text { constant }, \quad \dot{u}_{x}=\frac{P}{2 \eta}\left(h^{2}-z^{2}\right) .
$$

In the above equations, $h$ is the crack opening. Hence, the surface force due to viscosity is given by the formula:

$$
\eta \frac{\partial p}{\partial x}=-h P
$$

The volume force acting in any point of the complex structured continuum is given by:

$$
\sigma_{0} \eta \frac{\partial p}{\partial x}=-\sigma_{0} h P=-\frac{2 \sigma_{0}}{h} \eta<u_{x}>
$$

In the above equation $\left\langle u_{x}>\right.$ is the average particle velocity between two planes. It means, that the Navier-Stokes equation contains an additional term that describes the volume dissipative force against the fluid velocity, namely:

$$
\left.<F_{\tau}>=-k^{2} \dot{u}_{r}, \quad\left(k^{2}=2 \frac{\sigma_{0}}{h}\right)=\frac{\sigma_{0}^{2}}{f}\right),
$$

where $f$ is porosity.

\section{Stress in solid near borehole}

For the solid near borehole there are equations of equilibrium in cylindrical coordinates in the form:

$$
\begin{gathered}
\frac{\partial \sigma_{z z}}{\partial z}+\frac{\partial \tau_{r z}}{\partial z}+\frac{\tau_{r z}}{r}=\rho g \\
\frac{\partial \sigma_{r r}}{\partial r}+\frac{\sigma_{r r}-\sigma_{\varphi \varphi}}{r}+\frac{\partial \tau_{r z}}{\partial z}=0 .
\end{gathered}
$$

The stress pattern corresponding to the cylindrical coordinates are shown in Figure 5.

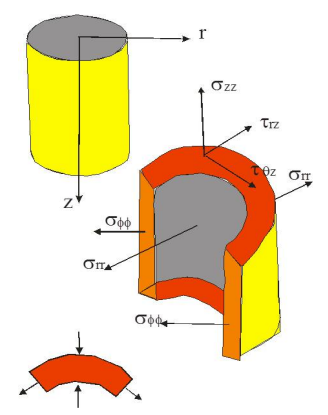

Figure 5: Stress nomenclature in cylindrical coordinates, and the representation of the borehole with liquid, and solid around it.

The above equations have very simple solutions, because for very large layer thickness in comparison to the radius of the borehole, the tangent stress $\tau_{r z}$ is equal to zero in all volume of rocks near the borehole. There is a known solution for the radial deformation $(e)$ of an elastic medium of density $\rho$ with a cylindrical cavity of radius $r_{0}$, and saturated by a liquid of density $\rho_{0}$ given by:

$$
e_{\varphi \varphi}\left(r_{0}\right)=\frac{u_{r}\left(r_{0}\right)}{r_{0}}=\rho g z\left(\frac{\sigma}{1-\sigma}-\frac{\rho_{0}}{\rho}\right) .
$$

In the above equation $\sigma$ is the Poisson ratio, $u_{r}$ is the radial displacement, and $g$ the gravity. Besides that, considering that there is planar incompressibility (no change in density with respect to the $z$ coordinate, compression along $z$, dilation along $x, y$, dilation along $z$, compression along $x, y$ ) in solid, we write that:

$$
e_{r r}+e_{\varphi \varphi}=0
$$


The respective solutions of equations (25) and (26) for the elastic stresses near borehole is given by the formulas (Sibiryakov, 1993):

$$
\begin{gathered}
\sigma_{r r}=\left(1-2 \gamma^{2}\right) P_{0}-\left(\frac{r_{0}}{r}\right)^{2} P_{0} \eta, \\
\sigma_{\varphi \varphi}=\left(1-2 \gamma^{2}\right) P_{0}+\left(\frac{r_{0}}{r}\right)^{2} P_{0} \eta .
\end{gathered}
$$

In equations (29) and (30), $\gamma=\frac{V_{S}}{V_{P}}, P_{0}$ is the weight of overburden rocks, and $\eta=1-2 \gamma^{2}-\frac{\rho_{0}}{\rho}$.

\section{Equation of equilibrium and velocity of fluid}

The equation of equilibrium with dissipative force around the borehole takes a form:

$$
\Delta \dot{u}_{r}-k^{2} \dot{u}_{r}-\frac{1}{\eta} \frac{\partial p}{\partial r}=0
$$

where $\dot{u}_{r}$ is the particle fluid velocity in the borehole. The normalized components are; the first term $\left(\Delta \dot{u}_{r}\right)$ the volume force, the second term $\left(-k^{2} \dot{u}_{r}\right)$ the volume dissipative force, and the third term $\left(-\frac{1}{\eta} \frac{\partial p}{\partial r}\right)$ the volume force due to pressure.

To find a solution for the above equation, a suggestion is that there is planar incompressibility for liquid as well as for solid, and that there is a linear relationship with respect to the vertical $z$ coordinate, like in (25). It means that there is a term $\frac{\partial^{2} \dot{u}}{\partial^{2} z^{2}}=0$ in Laplace operator, such that this operator simplifies to the form:

$$
\Delta \dot{u}_{r}=\frac{\partial^{2} \dot{u}_{r}}{\partial r^{2}}+\frac{1}{r} \frac{\partial \dot{u}_{r}}{\partial r} .
$$

With the assumption of planar (not in volume) incompressibility, there is additional conditions for equation (32) in the form:

$$
\frac{\partial \dot{u}_{r}}{\partial r}+\frac{\dot{u}_{r}}{r}=0, \quad \dot{u}_{r}=\frac{C(r)}{r^{2}} .
$$

where $C(z)$ is an arbitrary function. Besides that, we can write equation (32) in the form:

$$
\frac{\partial^{2} \dot{u}_{r}}{\partial r^{2}}+\frac{1}{r} \frac{\partial \dot{u}_{r}}{\partial r}=\frac{\partial}{\partial r}\left(\frac{\partial \dot{u}_{r}}{\partial r}+\frac{\dot{u}_{r}}{r}\right)+\frac{\dot{u}_{r}}{r^{2}}=\frac{\dot{u}_{r}}{r^{2}} .
$$

Substituting (34) into equation (32) gives a simpler expression for equation (31) in the form:

$$
\frac{\partial p(r)}{\partial r}=\eta\left(\frac{C(z)}{r^{3}}-k^{2} \dot{u}_{r}\right)
$$

Integrating the above equation with respect to $r$, with inferior limit $r$ and superior $R$, follows that:

$$
p(r)=P_{\infty}-\eta\left(\frac{C(z)}{2 r^{2}}-k^{2} C(z) \ln \frac{R}{r}\right) .
$$

To take into account that on the borehole surface $r=r_{0}$, $p \rightarrow P_{0}, R>>r_{0}$ and $p(R)=P_{\infty}$, we obtain:

$$
p\left(r_{0}\right)=P_{0}=P_{\infty}-\eta\left(\frac{\dot{u}_{0}}{2 r_{0}}+k^{2} \dot{u}_{0} r_{0} \ln \frac{R}{r_{0}}\right) .
$$

In the above equation $R$ is the horizontal range of the productive layer, $P_{\infty}$ is the liquid pressure outside the borehole (very far from it), and $P_{0}$ is the borehole liquid pressure. From formula (37), we obtain the velocity of fluid, $\left[\dot{u}_{0}\left(r_{0}\right)\right]$, into borehole, also with a convenient approximation

$$
\dot{u}_{0}\left(r_{0}\right)=\frac{P_{\infty}-P_{0}}{\eta} \frac{2 r_{0}}{1+2 k^{2} r_{0}^{2} \ln \frac{R}{r_{0}}} \approx \frac{P_{\infty}-P_{0}}{\eta} \frac{1}{r_{0} \ln \frac{R}{r_{0}}} \frac{f}{\sigma_{0}^{2}}=K \frac{f}{\sigma_{0}} .
$$

We simplified to the last form to emphasize the dependence of $f, \sigma_{0}$, and its ratio $\frac{f}{\sigma_{0}}$, with $K$ representing constant term. Figure 6 shows the plot of $\dot{u}_{0}\left(r_{0}\right)$ versus specific surface area, $\sigma_{0}$, and porosity, $f$, where the quantity $\frac{f}{\sigma_{0}}$ plays the role of permeability. This figure shows the rapid decay of $\dot{u}_{0}\left(r_{0}\right)$ versus $f$ and $\sigma_{0}$. Due to large specific surface area parameter, there is almost zero velocity flow inspite of sufficient porosity.

Also, for positive pressure contrast, $+\left|P_{\infty}-P_{0}\right|$, the flow is from layer reservatory to borehole, and when negative, $-\left|P_{\infty}-P_{0}\right|$, from borehole to layer.

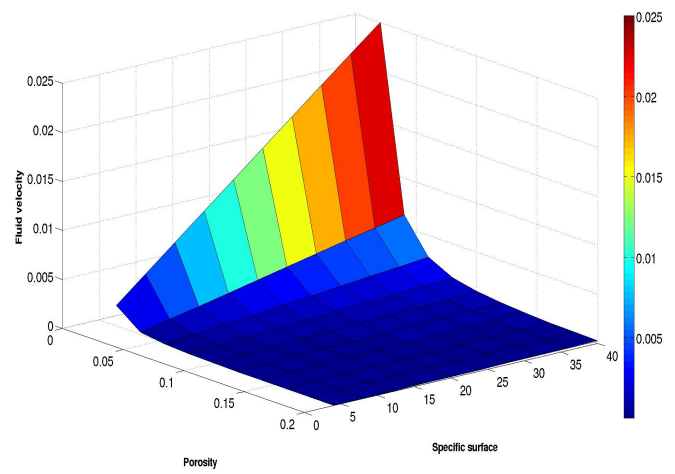

Figure 6: Plot of the formula of velocity flow (38) as a function of porosity, $f$, and specific surface area, $\sigma_{0}$.

It is interesting to mention that there is no necessity to use Darcy's law. Besides that, formula (38) does not contain the permeability coefficient, $k$. However, there is empiric relation between specific surface, $\sigma_{0}$, porosity, $f$, and permeability, $k$, namely (Sibiryakov and Prilous, 2007):

$$
k=\frac{D_{p}^{2} f^{3}}{150(1-f)^{2}}, \quad D_{p}=\frac{6}{\sigma_{0}}=\frac{6}{\frac{S_{E}}{V_{T}}},
$$

where $S_{E}$ is the total area of the porous space, and $V_{T}$ is the total volume of the sample, and $\sigma_{0}=\frac{S_{E}}{V_{T}}$. The equation (39) can be rewritten as:

$$
\sigma_{0}=\frac{\sqrt{6}}{5} \frac{f^{\frac{3}{2}}}{(1-f)} \frac{1}{\sqrt{k}} .
$$

The percolation process depends on porosity and specific surface only.

\section{Pressure in liquid far from borehole}

The value $P_{\infty}$ contained in equation (38) can be determined by solving of a problem about discontinuity between pressures in solid and in liquid. The usual stress-strain condition is given by the integration of the equation (14) of equilibrium, rewritten below:

$$
\frac{\partial \sigma_{i k}}{\partial x_{k}}=\rho g_{i}
$$


For simply stress conditions, when the vertical stresses are equal to the weight of rocks, but the horizontal stresses contain a factor of the type $\frac{\sigma}{1-\sigma},(\sigma$ is the Poisson coefficient), it is possible to formulate the following problem related to Figure 7: the elementary cube undergoes by

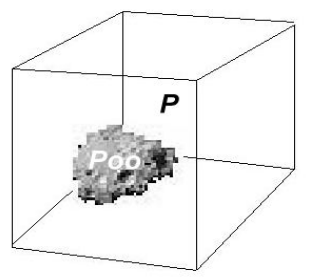

Figure 7: A cube volume where the liquid has pressure $P_{\infty}$, and the solid $P$.

vertical displacement $u_{0}$, while horizontal displacements are equal to zero. Inside the cube there is a liquid with constant pressure $P_{\infty}$. On the boundary solid-liquid the average normal displacement is given by the formula:

$$
<u_{n}>=\frac{P_{\infty}}{\rho_{0} c_{0}^{2}} \frac{f}{\sigma_{0}} .
$$

Under the condition of fluid incompressibility, the average tangent displacement on the boundary solid-liquid, it can be shown that:

$$
<\frac{\partial u_{S}}{\partial n}>=-\frac{<u_{n}>}{\pi l_{0}} .
$$

with $l_{0}$ as a dominant distance between porous. This result comes from differential geometry, and the quantities $\left\langle u_{n}\right\rangle$ and $\left\langle\frac{\left\langle u_{n}\right\rangle}{\pi l_{0}}>\right.$ have opposite sign due to physical counter action at the boundary solid-liquid.

The relation between $\sigma_{0}, l_{0}$ and $f$ is described by Sibiryakov and Prilous (2007), and it is given by:

$$
\sigma_{0} l_{0}=4(1-f)
$$

The simplification of boundary conditions as (42) and (43) gives the possibility to determine all stresses and displacements as a function of unknown constant $P_{\infty}$. On the vertical sides of the cube there are displacements equal to constant value, but on the other side it is equal to zero.

\section{Conclusions}

Comparison of the maps of intensity of tangential stresses $J(x, y, z)$ and of inclinations $\phi(x, y, z)$ of stress-produced fractures (see Figures 2 and 3 ) leads to the following conclusions: (1) faults detected from 3D seismic data correspond to local anomalies of tangential stresses; (2) the observed mismatch can be related to the difference between the present-day stress and the past stress along the faults; (3) different OWC depths in boreholes with numbers 124, 136, 139 can be caused by their position in different fluid-stress zones.

Porosity and specific surface area give a possibility to use alternative methods for measuring permeability.

The solution shows that the main factor of oil production is the specific surface area of porous and crack media. The solution does not contain the permeability; instead, there is the inverse square of specific surface area that plays the role of permeability.

The hydrofracture effect is due to a very small specific surface for several large fractures, in comparison to a lot of small fractures.

\section{Acknowledgments}

The authors would like to thank the Project INCT-GP, and in special to the Project Science Without Borders of CNPq/CAPES of the Brazilian Federal Government that sponsors this research aiming at oil exploration.

\section{References}

Landau, L. D., 1988, Hydrodynamics (in russian): Nauka. Moscow, Russia.

Novacky, W., 1970, Elasticity theory: Warsaw, Poland.

Sibiryakov, B. P., \& Prilous, B. I., 2007, The unusual small wave velocities in structural bodies and instability of pore or cracked media by small vibration: WSEAS Transactions on Applied and Theoretical Mechanics, 2, no. 7, 139-144.

Sibiryakov, B. P., \& Zaikin, A. D., 1994, Multiple seismics and applied geodynamics in petroleum-bearing regions: Geologiya i Geofizika (Russian Geology and Geophysics), 35, no. 5, 49-55.

Sibiryakov, B. P., Sibiryakov, E. B., Glebov, A. F., Nesterov, V. N., \& Sokolov, E. P., 2004, Prediction of stress and hydrodynamic behavior of fluids from multicomponent seismic data: Russian Geology and Geophysics, 45, no. 6, 752-759.

Sibiryakov, B. P., 1993, The stress-strain situation of rocks in vicinity of borehole: Russian Geology and Geophysics, 34, no. 6, 73-80.

Sibiryakov, B. P., 2002, Supersonic and intersonic cracking in rock-like material under remote stresses: Theoretical and Applied Fracture Mechanics, 38, no. 3, 255-265.

Sibiryakov, B. P., 2004, Dynamics of microinhomogeneous geological media: Novosibirsk State University, Russia. 